\title{
Precise and Accurate Determination of 54 Major, Minor and Trace Elements in Silicates Using a Standard Single LA-ICP- QMS
}

\section{ZHONGXING CHEN* AND CHARLES H. LANGMUIR}

Department of Earth and Planetary Sciences, Harvard

University, 20 Oxford Street, Cambridge, MA 02138, USA

E-mail: zchen@fas.harvard.edu

Despite many developments over three decades two main challenges have persisted for LA-ICPMS analysis: matrixdependent results and elemental fractionation. To address matrix dependence substantial efforts have been made to produce matrix matched standards, along with major efforts to reduce elemental fractionation largely through improvements in laser instrumentation. The latest femtosecond lasers, however, are prohibitively expensive for many users and elemental fractionation persists. Here we present analytical methods that eliminate the effects of both matrix dependence and elemental fractionation, improving data precision and accuracy as well as removing the need for matrix-matched standards. These methods then permit acquisition of data of high precision and accuracy for a large range of compositions. Using Thermo-Fisher Scientific Kinetic Energy Discrimination (KED) collision cell technology, all major and minor elements can be determined precisely and accurately on homogeneous silicate glass compositions on an anhydrous basis even without known internal standard concentrations. Normalizing to a fixed sum leads to excellent agreement with microprobe analyses $(<2 \%)$, as well as improved data quality for many elements (Na, K, P Mn), eliminating the need for a separate determination of internal standard elements by other analytical techniques. The net result leads to data that are a factor of 2-5 times improved relative to published work. Using a new encapsulated technique with a strip furnace, silicate powders can be rapidly converted to homogeneous glasses with little loss of volatile elements, permitting this approach to be used for all rocks without the need for chemical dissolution. This eliminates the problems with granitic rocks of trace phases that are resistant to acid dissolution. In combination these methods permit a single, rapid method for determination of a large suite of major, minor and trace element composition of silicates. Use of a single instrument leads to large savings in time and expense relative to conventional methods. Precision for a MORB glass run 50 times over six months shows $<2 \%$ RSD for $>5 \mathrm{ppm}$ and $<4 \%$ RSD for $>1 \mathrm{ppm}$. Similar precision and good accuracy are also documented for six micro-analytical geological glass reference materials with a wide variety of silicate matrices for 54 major, minor and trace elements. 